\title{
The L1 stalk is required for efficient export of nascent large ribosomal subunits in yeast
}

\author{
SHARMISHTHA MUSALGAONKAR, JOSHUA J. BLACK, and ARLEN W. JOHNSON \\ Department of Molecular Biosciences, The University of Texas at Austin, Austin, Texas 78712, USA
}

\begin{abstract}
The ribosomal protein Rpl1 (uL1 in universal nomenclature) is essential in yeast and constitutes part of the L1 stalk which interacts with E site ligands on the ribosome. Structural studies of nascent pre-60S complexes in yeast have shown that a domain of the Crm1-dependent nuclear export adapter Nmd3, binds in the E site and interacts with Rpl1, inducing closure of the L1 stalk. Based on this observation, we decided to reinvestigate the role of the L1 stalk in nuclear export of pre-60S subunits despite previous work showing that Rpl1-deficient ribosomes are exported from the nucleus and engage in translation. Large cargoes, such as ribosomal subunits, require multiple export factors to facilitate their transport through the nuclear pore complex. Here, we show that pre-60S subunits lacking Rpl1 or truncated for the RNA of the L1 stalk are exported inefficiently. Surprisingly, this is not due to a measurable defect in the recruitment of Nmd3 but appears to result from inefficient recruitment of the Mex67-Mtr2 heterodimer.
\end{abstract}

Keywords: L1 stalk; Mex67-Mtr2; export; ribosome

\section{INTRODUCTION}

Ribosomes are universally composed of one large and one small subunit, that function together to synthesize all proteins in a cell. Production of balanced levels of ribosomal subunits is critical for maintaining homeostasis in cells. In yeast four rRNA molecules and about 80 ribosomal proteins interact with more than 200 trans-acting assembly factors to achieve the complex task of ribosome synthesis (Woolford and Baserga 2013). Synthesis of new ribosomes by cells is a challenging and energy-consuming task and requires the coordinated expression of all ribosomal proteins and rRNAs. Failure to establish balanced expression levels of ribosomal proteins has been reported to cause cellular stress (Boulon et al. 2010; Cheng et al. 2019). Haploinsufficiency and mutations in ribosomal proteins in drosophila and zebrafish cause defects and delays in development (Amsterdam et al. 2004). In humans, mutations in genes coding for ribosomal proteins and biogenesis factors, give rise to a special class of diseases called ribosomopathies (De Keersmaecker et al. 2013; Mills and Green 2017; Warren 2018).

In eukaryotic cells, ribosome assembly starts in the subnuclear compartment called the nucleolus; it continues in the nucleoplasm followed by nuclear export; and concludes in the cytoplasm rendering fully matured subunits

Corresponding author: arlen@austin.utexas.edu

Article is online at http://www.rnajournal.org/cgi/doi/10.1261/rna. 071811.119. (for reviews, see Woolford and Baserga 2013; Kressler et al. 2017; Peña et al. 2017). Although the precursor rRNA for both subunits is a single transcript, RNA processing in the nucleolus separates precursors for the two subunits before nuclear export. Much of ribosomal subunit assembly is completed in the nucleus before the subunits are exported to the cytoplasm. Therefore, the nuclear import machinery has to undertake a critical task of escorting the highly hydrophilic and bulky preribosomal subunits through a hydrophobic environment of the nuclear pore complex (NPC). It was previously shown that large cargoes require multiple receptor molecules for effecting the transient and reversible collapse of the hydrophobic permeability barrier in NPC for rapid translocation (Ribbeck and Görlich 2002). Consistent with this model, several export factors are required for the export of nascent 605 subunits. Export is facilitated by the export adaptor protein $\mathrm{Nmd} 3$ that utilizes its nuclear export sequence (NES) to recruit the export-receptor $\mathrm{Crm} 1$ (Ho et al. 2000; Gadal et al. 2001). Nuclear export is also assisted by other noncanonical export factors including Arx1 (Bradatsch et al. 2007; Hung et al. 2008; Greber et al. 2012, 2016) and Bud20 (Altvater et al. 2012; Bassler et al. 2012) and the mRNA

(C) 2019 Musalgaonkar et al. This article is distributed exclusively by the RNA Society for the first 12 months after the full-issue publication date (see http://rnajournal.cshlp.org/site/misc/terms.xhtml). After 12 months, it is available under a Creative Commons License (Attribution-NonCommercial 4.0 International), as described at http:// creativecommons.org/licenses/by-nc/4.0/. 
export factor Mex67-Mtr2 heterodimer (Yao et al. 2007) in yeast but only $\mathrm{Nmd} 3$ appears to be conserved throughout eukaryotes as a dedicated 60S export factor (Thomas and Kutay 2003). However, unlike its essential role in 605 biogenesis, $\mathrm{Nmd} 3$ interaction with $\mathrm{Crm} 1$ is dispensable if other export receptors are fused directly to $\mathrm{Nmd} 3$ (Lo and Johnson 2009) indicating a general requirement for interaction with the NPC but not a specific requirement for a particular export receptor.

Recent high resolution structures of ribosome assembly intermediates have revealed the binding sites of numerous biogenesis factors including all known export factors with the exception of Mex67-Mtr2 (Matsuo et al. 2014; BarrioGarcia et al. 2016; Greber et al. 2016; Wu et al. 2016; Ma et al. 2017; Malyutin et al. 2017; Zhou et al. 2019). Arx1 binds on the solvent-exposed surface of the subunit near the peptide exit tunnel (PET) whereas Nmd3 binds on the subunit interface spanning the $E, P$, and A-sites of the subunit. However, the carboxy-terminal region of $\mathrm{Nmd} 3$ which contains the NES that recruits $\mathrm{Crm} 1$ is not resolved on any of the structures. Therefore, the position of $\mathrm{Crm} 1$ relative to the subunit during export is still unknown. Bud20 also binds on the subunit interface where it interacts with Tif6 and Rlp24, although the mechanism by which Bud20 promotes export is disputed (Altvater et al. 2012; Bassler et al. 2012). Densities for Mex67-Mtr2 heterodimer were not detected in any structures of pre-60S particles to date. However, UVinduced protein-RNA crosslinking studies in vivo identified crosslinks to many regions of the $25 \mathrm{~S}$ rRNA, but strongly enriched in the $3^{\prime}$ terminal end of 5.8S rRNA (Tuck and Tollervey 2013). In addition, a recent study attempting to reconstitute Mex67-Mtr2 binding to affinity-purified pre-60S particles in vitro identified crosslinks to $5.8 \mathrm{~S}$ and P-stalk rRNA (Sarkar et al. 2016). However, binding to the P-stalk was only observed for Yvh1-containing pre-60S particles while our recent structural studies and work from others show that Yvh1 joins the subunit only in the cytoplasm (Kemmler et al. 2009; Lo et al. 2009; Nerurkar et al. 2018; Zhou et al. 2019) and hence, cannot be responsible for promoting Mex67-Mtr2 binding to pre-60S in the nucleus.

Ribosomal Protein L1 (Rpl1) is a universally conserved protein which interacts with a single loop of the $25 \mathrm{~S}$ rRNA to form the L1 stalk. Rpl1 is essential in yeast and is encoded by two paralogous genes RPL1A and RPL1B. It was previously reported that 605 subunits lacking Rpl1 are exported to the cytoplasm and even detected in the polysomes (Mclntosh et al. 2011), suggesting that 605 assembly and export can proceed in the absence of Rpl1. However, the essential export adapter $\mathrm{Nmd} 3$ binds Rpl1 and facilitates closure of the L1 stalk (Ma et al. 2017; Malyutin et al. 2017; Zhou et al. 2019). Because of the interaction between Rpl1 and Nmd3, we suspected that ribosomes lacking Rpl1 would be affected in their ability to bind $\mathrm{Nmd} 3$ or to release it.
Here, we show that Rpl1 protein is needed for efficient nuclear export of nascent large subunits precursors. The repression of the RPL1 or truncation of the L1 stalk rRNA reduced the efficiency of export but did not completely block export from the nucleus. Nascent subunits lacking Rpl1 maintained binding to the export factors $\mathrm{Nmd3}$, Arx1, and Bud20 but only inefficiently recruited the Mex67-Mtr2 heterodimer. Cooverexpression of MEX67 and MTR2 in RPL1 repressed cells overcame the $60 \mathrm{~S}$ export defect caused by loss of Rpl1 suggesting that delayed export of Rpl1-deficient subunits is due to a failure in Mex67-Mtr2 recruitment.

\section{RESULTS}

\section{Repression of Rpl1 leads to a 60S subunit export defect}

Recent cryo-EM structures of the nuclear export adapter $\mathrm{Nmd} 3$ on the $60 \mathrm{~S}$ subunit revealed a large interface between the elF5A-like domain of $\mathrm{Nmd} 3$ and ribosomal protein Rpl1 on the L1 stalk (Ma et al. 2017; Malyutin et al. 2017; Kargas et al. 2019; Zhou et al. 2019). The interaction between Nmd3 and Rpl1 holds the L1 stalk in a closed conformation in which the L1 stalk is bent toward the E site. Conceivably, the interaction between Rpl1 and Nmd3 may be important for the recruitment of $\mathrm{Nmd} 3$ to the pre-60S subunit in the nucleus. Alternatively, this compact structure could facilitate the export of the nascent $60 \mathrm{~S}$ subunit through the NPC or facilitate the release of Nmd3 from the pre-60S subunit after export to the cytoplasm. Although previous work reported that Rpl1 was not needed for 60S export (Mclntosh et al. 2011), we decided to revisit this question. We first asked if the nuclear export of $60 S$ subunits was affected by the loss of Rpl1. In yeast, Rpl1 is expressed from two paralogous genes, RPL1A and $R P L 1 B$, each encoding identical proteins. Because deletion of both genes is lethal, we used a conditional mutant in which RPL1B was deleted and RPL1A was under control of the galactose inducible/glucose repressible GAL1 promoter. 60S subunit localization was monitored with a GFP fusion to Rpl25. On shifting cells from galactose to glucose to repress Rpl1A expression, we observed a strong change in Rpl25-GFP localization from the cytoplasm to the nucleus (Fig. 1A), indicating impaired 60S export. Sik1-mRFP, expressed from a plasmid, was used as a nucleolar marker and it did not change its localization on repression of the RPL1 gene. Similar results were obtained using Rpl8-GFP as a reporter (data not shown). The accumulation was most evident within 60-90 min after returning saturated cultures to active growth. At longer time points, Rpl25 signal became increasingly cytoplasmic, indicating that export continued, albeit at a lower efficiency than when Rpl1A was expressed (data not shown). Nuclear accumulation of Rpl25-GFP was also observed in an 
A

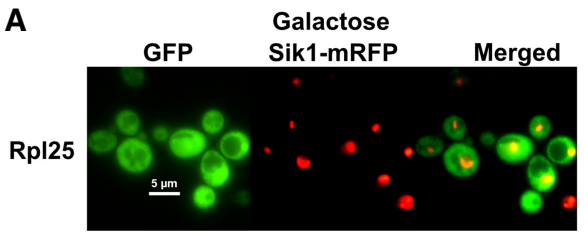

B
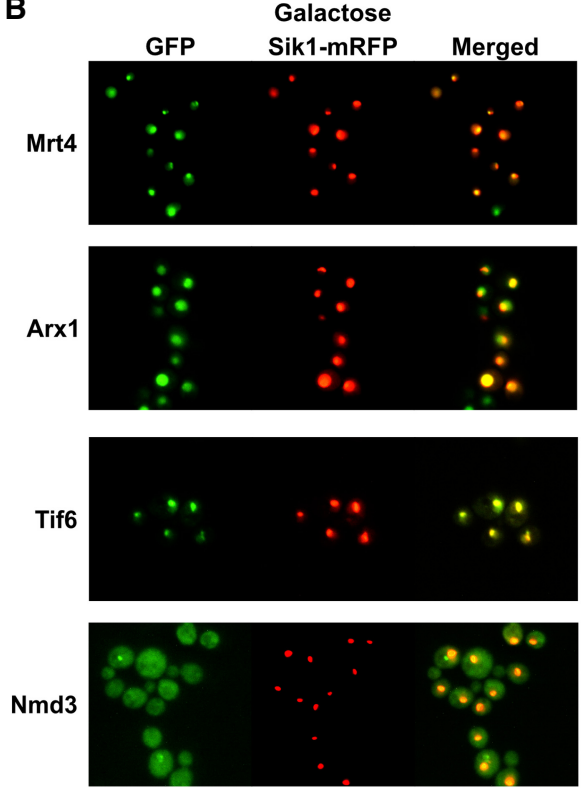
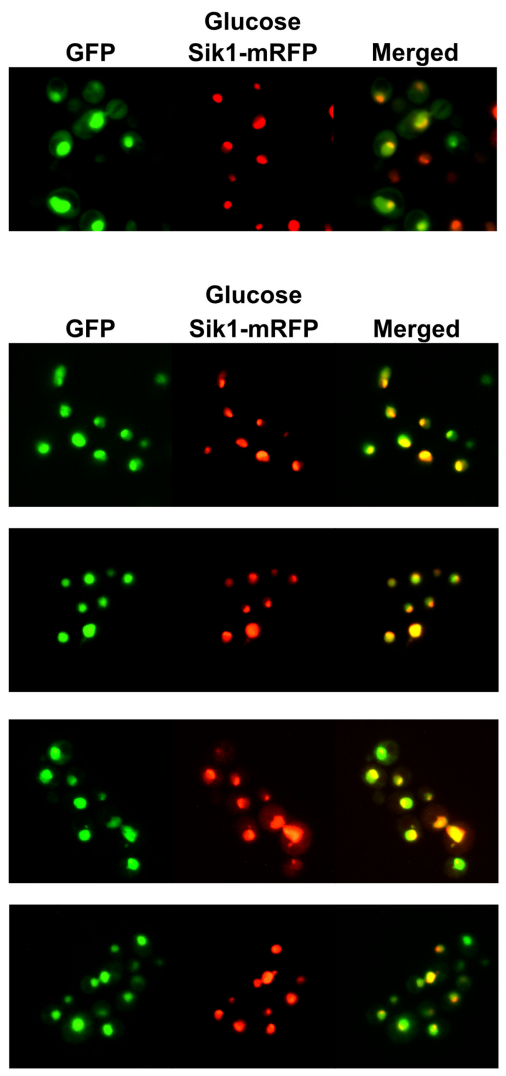

FIGURE 1. Depletion of Rpl1 reduces 60S export. (A) The localization of Rpl25-GFP expressed from plasmid pAJ907 and Sik1-mRFP expressed from pAJ4338 were visualized in cells of strain KBM20 expressing RPL1A (Galactose) or after $2 \mathrm{~h}$ of repression of RPL1A (Glucose). GFP, tagged Rpl25. (B) The localization of Mrt4-GFP (AJY3850), Tif6-GFP (AJY3848), Arx1-GFP (AJY3849), and Nmd3-GFP (AJY4060) and Sik1-mRFP expressed from pAJ4338 were visualized in cells expressing RPL1A (Galactose) or after $2 \mathrm{~h}$ of repression of RPL1A (Glucose). Sik1 was used as a marker for the nucleolus.

rp/1b4 strain in which Rpl1 was constitutively expressed at reduced levels compared with wild-type cells due to deletion of RPL1B (see above).

Pre-60S subunits are accompanied to the cytoplasm with a host of assembly factors including Nmd3, Mrt4, Tif6, and Arx1 (Barrio-Garcia et al. 2016; Wu et al. 2016; $\mathrm{Ma}$ et al. 2017; Zhou et al. 2019). Although each of these factors continually shuttles in and out of the nucleus, they show different steady-state localizations: Mrt4 and Tif6 are predominantly nucleolar whereas Arx 1 is nucleoplasmic and Nmd3 is cytoplasmic (Lo et al. 2010). On glucose repression of RPL1 expression, Mrt4 and Tif6 relocalized from the nucleolus to the nucleoplasm whereas the nucleoplasmic localization of Arx1 was largely unchanged (Fig. 1B). Strikingly, Nmd3 was relocalized from the cytoplasm to the nucleoplasm (Fig. 1B). These results imply that in the absence of Rpl1 expression, pre-60S particles containing Tif6, Mrt4, and probably Arx1 and Nmd3 accumulate at a late assembly step in the nucleoplasm, prior to export. We conclude that Rpl1 is necessary for efficient export of 605 subunits from the nucleus.

\section{Nmd3 binds to nascent subunits lacking Rpl1}

Because Nmd3 binds to Rpl1 and provides an essential Crm1-dependent nuclear export signal for the $60 \mathrm{~S}$ subunit, a lack of Nmd3 binding to the pre-60S particle could explain the export block observed on repression of Rpl1. We tested if Nmd3 is lost from nascent 605 subunits under conditions where we observed accumulation of $\mathrm{Nmd3}$ in the nucleus after Rpl1 repression. Cells expressing Rpl1 from its native promoters or under control of the glucose-repressible GAL1 promoter were shifted from galactose to glucose. Extracts were prepared and sedimented through sucrose density gradients and the positions of Nmd3, Rpl1, and Rpl8 were monitored by western blotting. Surprisingly, Nmd3 cosedimentation with free 605 subunits was largely unaffected by RPL 1 repression (Fig. 2, cf. B and A). The slight accumulation of free $\mathrm{Nmd} 3$ at the top of the gradient in Rpl1-repressed cells (Fig. 2B) cannot account for the bulk redistribution of $\mathrm{Nmd} 3$ from the cytoplasm to the nucleus in these cells. These results suggest that the population of $\mathrm{Nmd} 3$ that accumulates in the nucleus on RPL1 repression is bound to pre-60S subunits.

To test directly if Nmd3 binds to subunits lacking Rpl1, we immunoprecipitated subunits associated with $\mathrm{Nmd} 3$ from cells in which Rpl1 was expressed or repressed. We used Arx 1 as a control for a pre-60S associated protein whose binding is not expected to be dependent on Rpl1. Similar levels of 605 subunits, indicated by Rpl8, coimmunoprecipitated with $\mathrm{Nmd} 3$ and Arx 1 regardless of Rpl1 expression (Fig. 2C). However, the ratio of Rpl1 to Rpl8 in the immunoprecipitated samples was significantly reduced by when Rpl1 was repressed (Fig. 2D). These results show that $\mathrm{Nmd} 3$ can bind subunits lacking Rpl1, despite the loss of a large interaction surface between these two proteins.

\section{Truncation of the L1 stalk leads to a 605 export defect}

As a complementary means of assessing the importance of the L1 stalk for 60S export, we truncated the RNA of the L1 stalk. We replaced nucleotides 2451-2495 with the GNRA tetraloop GAGA (Correll et al. 1999; Ben-Shem et al. 

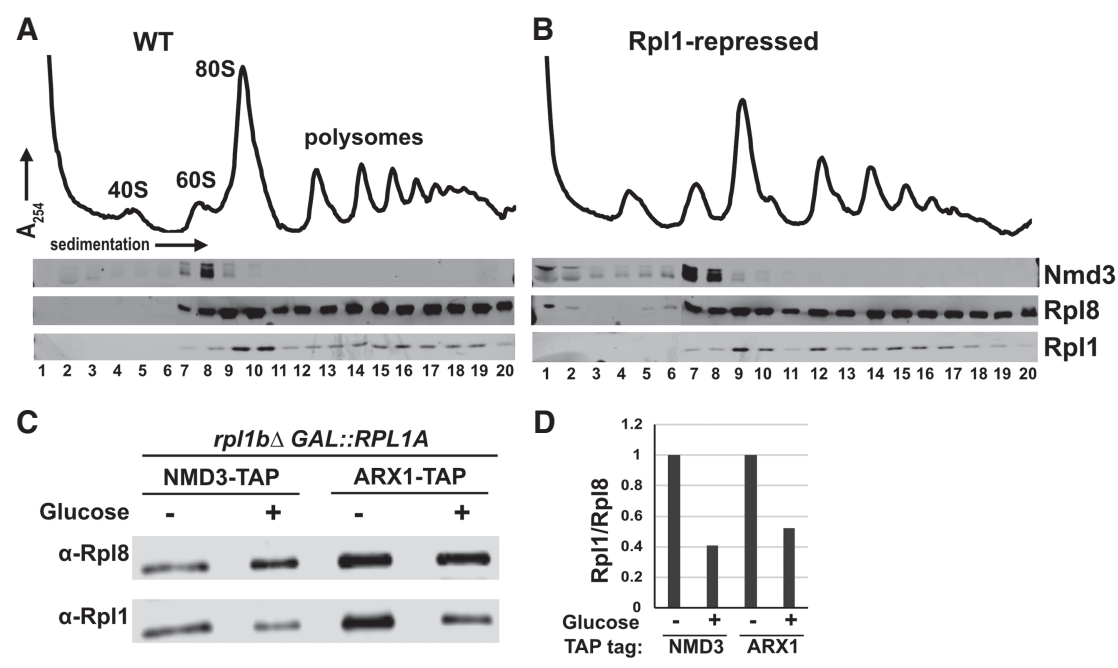

FIGURE 2. Nmd3 binds to subunits lacking Rpl1. (A), (B) Polysome profiles and western blots for monitoring sedimentation of Nmd3, Rpl1, and Rpl8 from extracts of WT (KBM13) and GAL:: RPL1 (KBM20) cells, respectively, grown in galactose media followed by $2 \mathrm{~h}$ growth after adding glucose. (C) Western blots for affinity purification of Nmd3-TAP (AJY4009, lanes 1,2) and Arx1-TAP (AJY4013, lanes 3,4) from GAL::RPL1 cells either grown in galactose medium continually (lanes 1,3) or for $2 \mathrm{~h}$ after addition of glucose (lanes 2,4). (D) Ratios of Rpl1 to Rpl8 signal from western blot in $C$ were calculated and normalized to the Rpl1 to Rpl8 ratios for cells grown continuously in galactose medium.

2011), deleting the entire Rpl1 binding site (Fig. 3A). We made the truncation in a construct that ectopically expressed 25S rRNA with a unique oligo tag to be able to monitor the mutant ribosomal RNA in the presence of wild-type 60S. The oligo tag was inserted in ES8 and had no discernible effect on function (Fig. 3B). As anticipated, truncation of the L1 stalk was lethal, shown by its inability to complement deletion of the genomic rDNA locus (Fig. 3B). Nevertheless, we were able to monitor localization and incorporation of the mutant rRNA into subunits using fluorescence in situ hybridization (FISH) and northern blotting, respectively. The RNA of the L1 stalk truncation mutant accumulated in the nucleus but could also be detected in the cytoplasm (Fig. 3C), indicating that subunits with a truncated L1 stalk, and hence lacking Rpl1, can be exported to the cytoplasm, albeit less efficiently than wild-type. Surprisingly, this RNA sedimented in 60S, 80S, and in polysomes, indicating that despite lacking a functional L1 stalk, the mutant RNA was incorporated into actively translating ribosomes. This was consistent with a previous report that Rpl1-deficient ribosomes can engage in translation (Mclntosh et al. 2011). However, comparison of the ratio $25 \mathrm{~S}$ rRNA from the L1 stalk $\Delta$ mutant to endogenously expressed $25 \mathrm{~S}$ rRNA revealed differences in sedimentation of the mutant RNA compared with WT. Notably, the mutant strongly accumulated in free 60S subunits (Fig. 3D, right panel, lanes 6,7) and was somewhat enriched over wild-type in 805 and light polysomes (fractions 10-15) but was relatively depleted from deep polysomes (fractions 16-19). A shift toward lighter polysomes sug- gests that ribosomes without a functional L1 stalk arrest at or shortly after translation initiation. Together, these results indicate that ribosomes with a truncated L1 stalk are exported less efficiently and engage with $40 \mathrm{~S}$ subunits but accumulate in light polysomes, possibly because they are defective for elongation.

\section{Pre-60S subunits without Rpl1 fail to recruit Mex67-Mtr2 heterodimer efficiently}

The accumulation of Rpl25 and various shuttling biogenesis factors in the nucleus suggested that nascent 605 subunits lacking Rpl1 were defective in nuclear export. Possibly, nascent subunits lacking Rpl1 were unable to recruit factors involved in 605 export because of structural differences caused by loss of Rpl1. To identify such factors, we affinitypurified nascent subunits and performed mass spectrometric proteomic analysis on them. After observing that $\mathrm{Nmd} 3$ can bind to large subunit particles lacking Rpl1 (Fig. 2A-C), we decided to use carboxyterminal TAP-tagged Nmd3 as a bait for affinity purifying Pre-60S particles in RPL1 repressed cells. As shown above, loss of Rpl1 from the pre-60S particle affected their nuclear export and accumulated particles in the nucleoplasm. For comparison, we affinity-purified Nmd3-TAP particles from cells treated with LMB in an LMB-sensitive CRM1T539C background (Grosshans et al. 2001), to mimic the nuclear accumulation of Rpl1-containing particles.

Spectral counts obtained from the mass spectrometric analysis of the eluted samples were used to generate relative spectral abundance factor (RSAF) values as described previously (Sardana et al. 2015). We then generated ratios for RSAF values for each protein in the sample to that of Tif6 protein in the same sample and normalized values to the L1-expressed + LMB samples. Figure 4A summarizes results from two independent experiments, comparing the relative RSAF values for the 60S export factors Arx1, Bud20, and Mex67. While depletion of Rpl1 had no effect on the association of Arx1 or Bud20 with Nmd3-bound pre-60S particles, Mex67 was not detectable on these particles (Fig. 4A). The loss of Mex67 from these particles was not due to reduced expression of Mex67 in the Rpl1-repressed cells (Fig. 4E).

To corroborate the results from the mass spectrometric, we also analyzed both the Rpl1-repressed, and the Rpl1containing and LMB-treated samples by SDS-PAGE and western blotting for Rpl8, Rpl1, and Mex67. For additional 
A

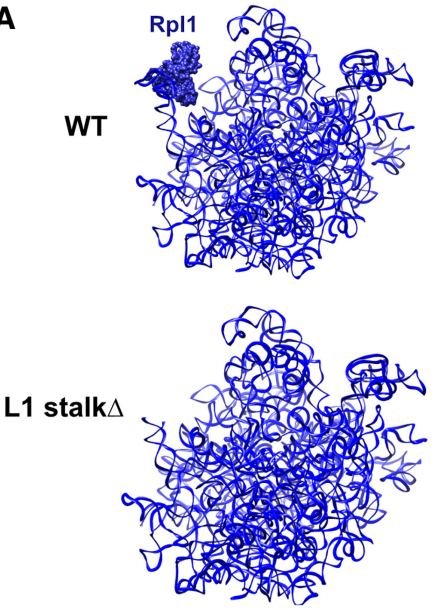

B
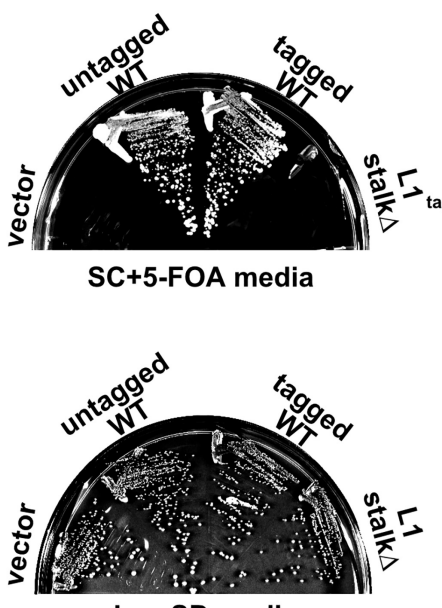

C
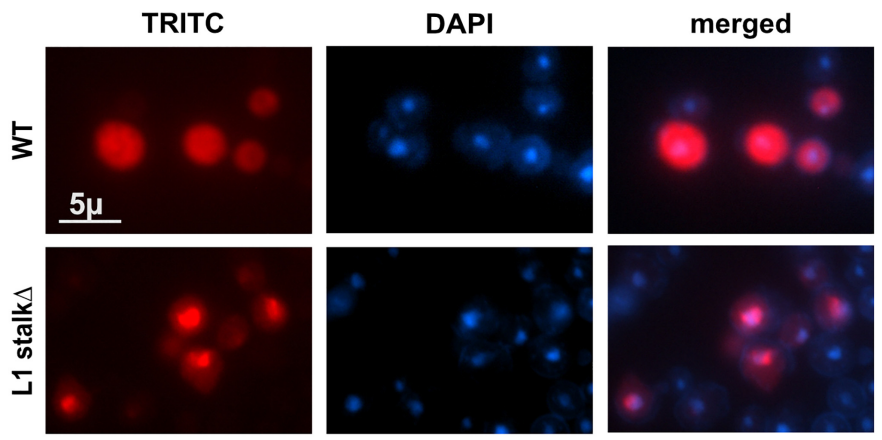

D
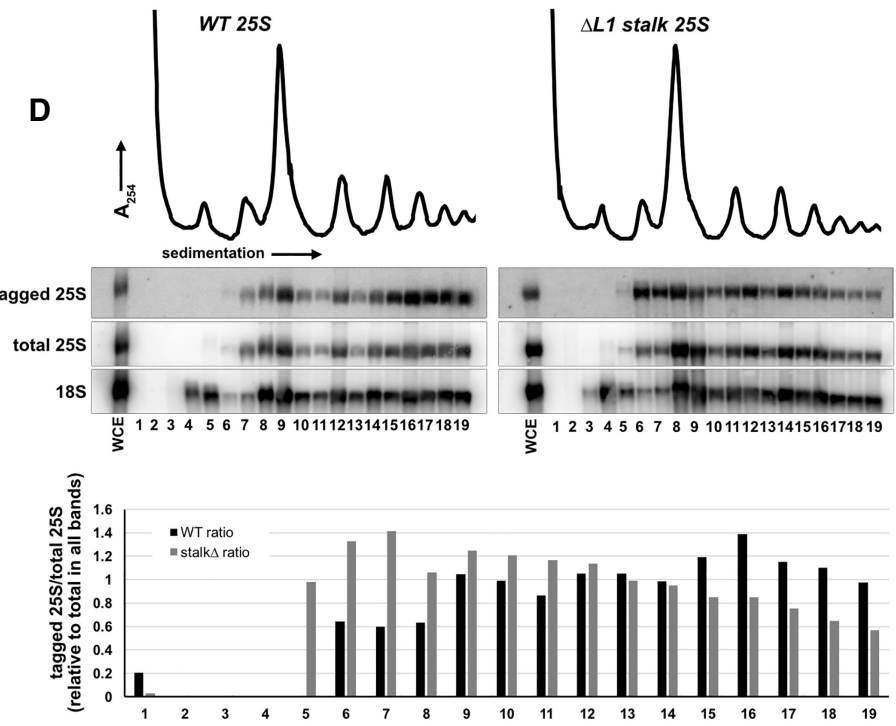

FIGURE 3. Truncation of L1 stalk RNA leads to a 60 S export defect. (A) Cartoon of $25 \mathrm{~S}$ rRNA for WT and L1 stalk truncation showing expected lack of Rpl1 binding when RNA was truncated. (B) Plasmids constructs expressing WT (pAJ1181) or L1 stalk $\Delta$ (pAJ3605) rRNA were transformed into AJY1185 (rDNA $\Delta, 35$ S URA3 2 $\mu$ ) and complementation was tested on 5-FOA media. (C) Fluorescence in situ hybridization and microscopy using oligo (AJO628) hybridizing to a unique tag in 25S rRNA expressed from plasmid constructs for WT (pAJ1181) and L1 stalk $\Delta$ (pAJ3605) in strain BY4741. (D) Sucrose gradient sedimentation and northern blot analysis using oligo (AJO628) against a unique tag on rRNA expressed from WT (pAJ1181) or L1 stalk $\Delta$ (pAJ3605) rRNA. Total 25S and 18S rRNAs were detected using oligos AJO192 and AJO190, respectively.

controls, we carried out mock TAP purification from untagged cells as well as Nmd3-TAP purification from wildtype and Rpl10-repressed cells, to trap particles after export and at a very late step in cytoplasmic maturation at which Mex67 would be expected to have already been released. Finally, as an additional control experiment, particles were purified using Arx1-TAP from WT or RPL1repressed cells. Similar to the mass spectrometric analysis, the amount of Mex67 coprecipitating with Nmd3-bound particles sharply decreased in RPL1-repressed cells compared with LMB-treated cells (Fig. 4B, lanes 2,3), suggesting that pre-60S particles devoid of Rpl1 inefficiently recruited Mex67. In samples from WT cells, without any LMB treatment (lane 4), relative Mex67 levels were comparable to those in the LMB-treated sample. The RPL10repressed sample also exhibited a sharp decrease in Mex67 levels (Fig. 4B, lane 5), as expected for a late- cytoplasmic particle. In Arx1-TAP samples too, less Mex67 was coprecipitated from RPL1-repressed cells compared with WT cells (Fig. 4B, lanes 6,7). However, the decrease was subtle compared with Nmd3-TAP particles perhaps because Arx1 binds pre-60S earlier than Nmd3, significantly and before Mex67 and hence a smaller population of Arx1 particles is bound to Mex67-containing particles.

We also tested the association of Mex67 with subunits lacking Rpl1 by sucrose gradient sedimentation. Extracts were prepared from RPL1-expressing or RPL1-repressed cells, separated on sucrose gradients and the sedimentation of Mex67 and Rpl8 was monitored by western blotting (Fig. 4C). In both conditions, Mex67 cosedimented at the $60 \mathrm{~S}$ peak in addition to the top of the gradient. Although more Mex67 cosedimented with free 60S in RPL1-repressed cells compared with nonrepressed cells, there was also a significant accumulation of free 605 
A

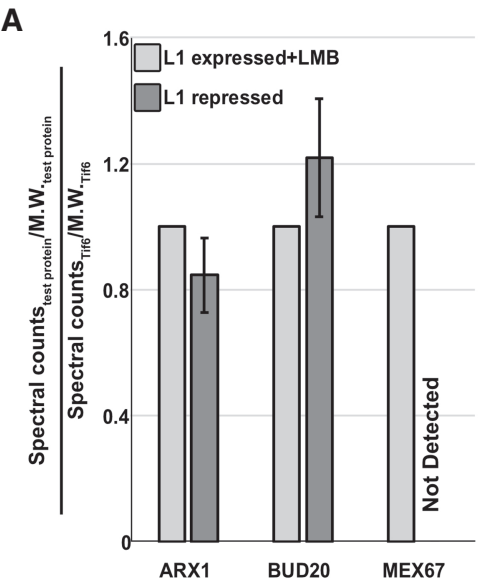

C

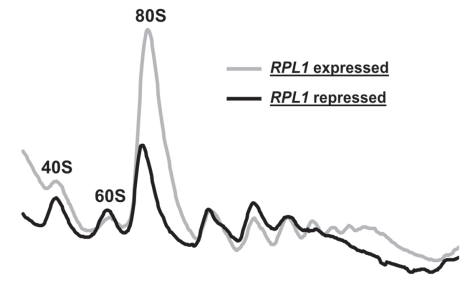

fraction \begin{tabular}{lllllllll} 
RPL1 expressed \\
\hline
\end{tabular}

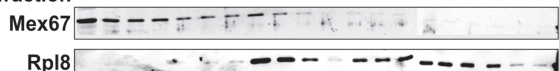

Rpl8

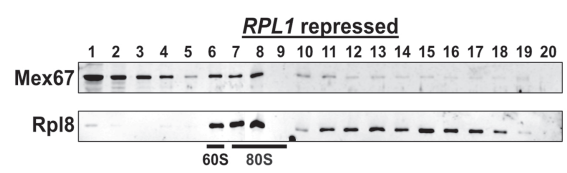

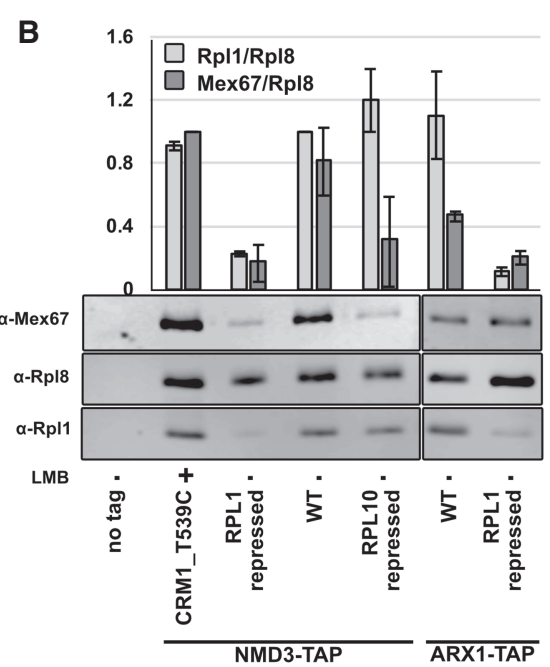

D

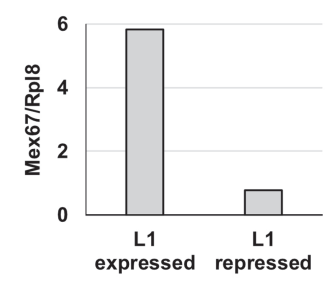

E

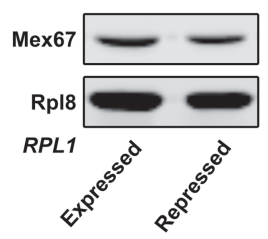

FIGURE 4. Nascent subunits lacking Rpl1 fail to recruit Mex67 efficiently. (A) Nmd3-TAP was purified from RPL1-repressed cells and from LMB-treated cells. Spectral counts/molecular weight (MW) for Arx1, Bud20, and Mex67 were normalized to Tif6 levels in each sample. TAP purifications were from AJY4008 treated with LMB for $30 \mathrm{~min}$ and from AJY4009 in which RPL1 was repressed for $1.5 \mathrm{~h}$. (B) Western blots for Mex67, Rpl1, and Rpl8 in TAP purification samples from BY4741, AJY4008 treated with LMB for 30 min, AJY4009, AJY1874, AJY4001, AJY4012, and AJY4013 grown in galactose followed by $1.5 \mathrm{~h}$ glucose treatment (lanes 1-7, respectively). Mex67:Rpl8 and Rpl1:Rpl8 were calculated for each sample. Mex67:Rpl8 ratio in each sample was normalized to that in the LMB sample (lane 2), and Rpl1:Rpl8 ratio in each sample was normalized to that in the WT NMD3-TAP samples (lane 4). (C) Polysome profiles and western blots for monitoring sedimentation of Mex67 and Rpl8 done in the low salt buffer $\left(50 \mathrm{mM} \mathrm{K}^{+}\right)$from extracts of WT (KBM13) and GAL::RPL1 (KBM20) cells, respectively, grown in galactose media followed by $2 \mathrm{~h}$ growth after adding glucose. (D) Mex67:Rpl8 in the fraction containing the 60S subunits was calculated for sucrose gradients of RPL1 expressed and repressed conditions in C. (E) Western blots for Mex67 and Rpl8 in extracts from KBM13 and KBM20 grown in galactose containing media for $48 \mathrm{~h}$ and then diluted and grown in fresh glucose-containing medium for $1.5 \mathrm{~h}$.

\section{High copy expression of the Mex67-Mtr2 heterodimer specifically suppresses the export defect of Rpl1 repression}

The low levels of nuclear export factor Mex67 associated with nascent 60S subunits purified from Rpl1-repressed cells suggested that Rpl1 may have a role in recruiting the Mex67-Mtr2 heterodimer to nuclear pre-60S. To test if the export block could be overcome, we cooverexpressed Mex67 and Mtr2 in rp/1b4 cells with RPL1A under galactose inducible/glucose repressible promotor also expressing Rpl25-GFP. As shown in Figure 1, Rpl25-GFP accumulated in the nucleus on repression of RPL1A but remained cytoplasmic under the same conditions in the WT cells (Fig. 5A). However, cooverexpression of Mex67 and Mtr2 alleviated the nuclear export defect of nascent subunits, monitored by Rpl25-GFP localization. To test if the effect of Mex67-Mtr2 overexpression was specific to these export factors, we asked if over-expressing other 605 nuclear export factors, Arx 1 and Bud20, could mitigate the nuclear export defect caused by Rpl1 loss. Overexpression of neither of these two proteins affected the nuclear localization of Rpl25, suggesting that the Mex67-Mtr2 binding is specifically affected on Rpl1 loss. Although overexpression of Mex67 and Mtr2 suppressed the nuclear export defect of Rpl1 repression, cooverexpression of Mex67 and Mtr2 did not suppress the lethality caused by repression of Rpl1 (Fig. 5B) or growth defect seen in rp/1b $\Delta$ strain (data not shown), as expected because Rpl1 is an essential ribosomal protein that interacts with $E$ site ligands during the translation cycle. subunits in these cells. In fact, Mex67 was underloaded on these subunits, evidenced by the low Mex67 to Rpl8 ratio (Fig. 4D). Because pre-60S accumulates in the nucleus when Rpl1 is repressed, these results indicate that Mex67 is underloaded on free 60S subunit that accumulates in the nucleus when Rpl1 is repressed.

\section{DISCUSSION}

Here, we have shown that the L1 stalk is needed for efficient export of pre-60S subunits from the nucleus. Although it was previously demonstrated that ribosomes lacking Rpl1 can engage in translation and, therefore, 


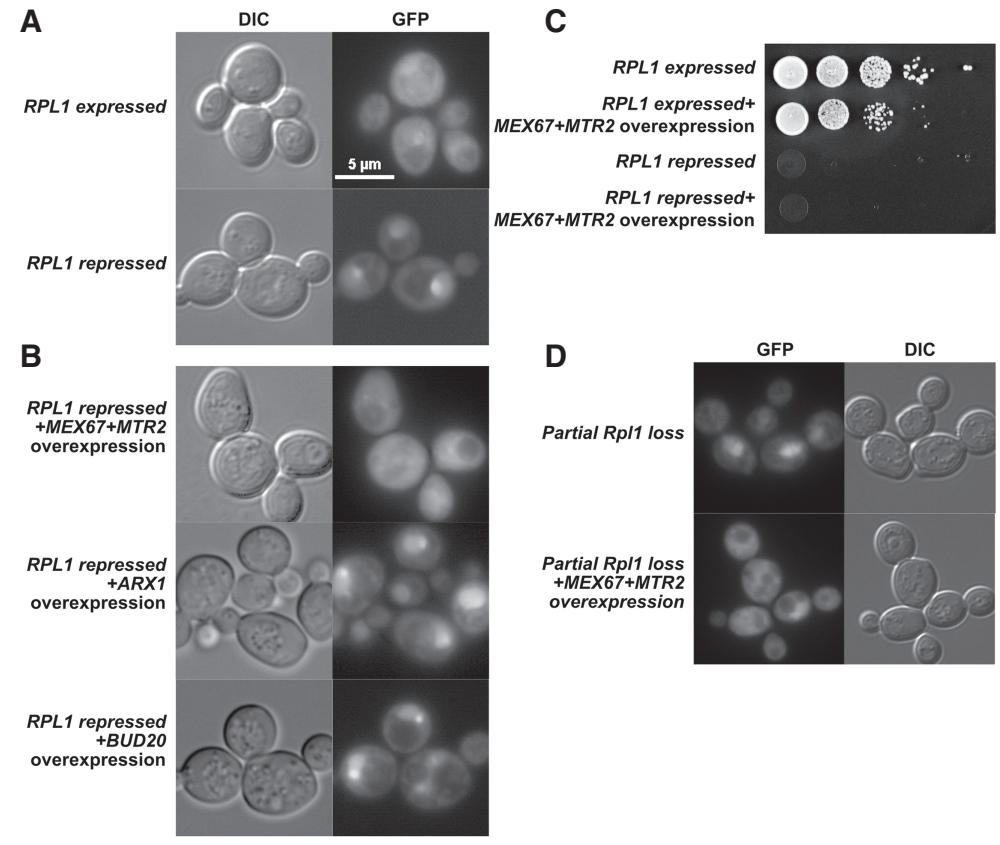

FIGURE 5. High copy expression of Mex67-Mtr2 heterodimer specifically suppresses the 60S export defect caused by Rpl1 loss. Rpl25-GFP viewed in (A) WT (KBM13) transformed with RPL25GFP (pAJ907) and empty vector (pRS426) and in rpl1b4 P GAL-RPL1A (KBM20) with RPL25-GFP and empty vector or (B) high copy MEX67+MTR2 (pAJ3972), ARX1 (pAJ4315), or BUD20 (pAJ4316) and grown in Leu-Ura-media with galactose for $48 \mathrm{~h}$ and then diluted fivefold in glucose-containing media and grown for $1.5 \mathrm{~h}$ more. $(C)$ Tenfold serial dilutions of the KBM13 or KBM2O transformed with empty vector or MEX67+MTR2 were spotted on glucose-containing selective media to repress $P_{G A L}: R P L 1 A$ in KBM20. (D) Rpl25-GFP viewed in the upper panel: rp/1 b4 (KBM17) transformed with RPL25-GFP (pAJ907) and empty vector (pRS426) and lowerpanel: KBM17 transformed with RPL25-GFP and MEX67 MTR2 and grown in Leu-Ura-media with glucose for $48 \mathrm{~h}$ and then diluted fivefold in glucose-containing media and grown for $1.5 \mathrm{~h}$ more. subunit for correct assembly, our work demonstrates that there is not a strict quality control pathway that assesses assembly of the L1 stalk. A similar observation was recently made for the RNA of internal transcribed spacer 2 (ITS2), which connects $5.8 \mathrm{~S}$ and $25 \mathrm{~S}$ RNA. Another study had previously reported that premature 60S particles containing 7S pre-rRNA can be exported to the cytoplasm and can even engage in translation (Rodríguez-Galán et al. 2015). The $232 \mathrm{nt}$ of ITS2 are normally removed by RNA processing in the nucleolus, prior to export. However, in mutants that are blocked for ITS2 processing, pre$60 \mathrm{~S}$ subunits retaining ITS2 RNA are exported to the cytoplasm (Sarkar et al. 2017). These ITS2-containing subunits also engage with $40 \mathrm{~S}$ subunits in translating ribosomes. However, they appear to induce translational stress and are recognized by cytoplasmic quality control pathways involving 3'-RNA decay machinery and the ROC complex. In both cases, whether the defective ribosomes themselves are targeted for degradation and/or induce degradation of associated mRNAs remains to be resolved. must be exported (Mclntosh et al. 2011; Shi et al. 2017; Segev and Gerst 2018), those studies did not explore a role for Rpl1 in nuclear export. Considering that Rpl1 is essential in yeast and provides part of the binding site for the nuclear export adapter Nmd3, it is somewhat surprising that loss of Rpl1 does not have a greater impact on 605 export. Nmd3 is conserved from archaea to higher eukaryotes, indicating that it has a more fundamental role in ribosome maturation that predates the evolution of the nuclear envelope. Whereas the euryarchaeal proteins are similar to eukaryotic $\mathrm{Nmd} 3$ and contain an elF5A-like domain which interacts with Rpl1, the lower archaeal $\mathrm{Nmd} 3$ proteins lack this domain. Thus, the conserved function of $\mathrm{Nmd3}$, which is likely to promote the loading of Rpl10 (uL16) (Zhou et al. 2019), is independent of Rpl1 binding. The interaction with Rpl1 appears to be a more recent evolutionary development and may not be essential for Nmd3 function.

\section{Quality control and the L1 stalk}

Despite the essential nature of the L1 stalk and the expectation that quality control mechanisms monitor the nascent

\section{What is the role of the L1 stalk in large subunit export?}

The L1 stalk could impact export by one of a couple different but not mutually exclusive mechanisms. Because the L1 stalk is a highly flexible hydrophilic appendage, it might be unfavorable for passage through the NPC. Closing the L1 stalk by binding to Nmd3 would present a more compact structure to facilitate export. Similarly, expansion segment 27 (ES27) forms a long dynamic helix in the vicinity of the exit tunnel and is captured by the export factor Arx1, restraining its movement (Greber et al. 2016). Conceivably, tethering both the L1 stalk and ES27 could be mechanisms to facilitate export. In an attempt to ask if reducing the length of the L1 stalk could enhance export by eliminating a "floppy" RNA element, we made further truncations of the L1 stalk. However, we did not observe enhanced export of these larger L1 stalk truncations (data not shown).

Alternatively, the L1 stalk may be important for efficient recruitment of an export factor. The translocation of large cargo molecules through the NPC requires multiple receptors (Ribbeck and Görlich 2002). Indeed, nuclear export of 
pre-60S subunits in yeast requires the export adapter Nmd3 (which recruits the receptor Crm1), the mRNA export-receptor Mex67-Mtr2 as well as Arx1 and Bud20 (Altvater et al. 2012; Bassler et al. 2012). Whereas the binding sites for Nmd3, Arx1, and Bud20 are wellestablished, the binding site for Mex67-Mtr2 has been enigmatic. Although the Mex67-Mtr2 duplex can bind $5 S$ rRNA (Yao et al. 2007), in vitro UV-induced crosslinking of Mex67 reconstituted with pre-60S particles affinity purified with Yvh1, identified binding sites in the $P$ stalk and 5.8S but not 5S rRNA (Sarkar et al. 2016). However, our recent structural analysis of pre-60S maturation (Zhou et al. 2019) shows that Yvh1 is recruited to the pre-60S only after Nog1 is released in the cytoplasm, a conclusion reached by others as well (Nerurkar et al. 2018). Thus, Yvh1 loads onto the pre-60S particle after export from the nucleus, and after the requirement for Mex67 in export. We suggest that in the Yvh1-bound particle, P stalk RNA is exposed due to the absence of either Mrt4 or the stalk protein PO, possibly offering a site for promiscuous binding by Mex67-Mtr2. UV-induced crosslinking of Mex67 to RNAs in vivo identified a wide distribution of crosslinking sites in $25 \mathrm{~S}$ and 5.8S rRNA (Tuck and Tollervey 2013) with strong hits in 5.8S, overlapping what was found in vitro (Sarkar et al. 2016). In neither of these crosslinking studies was Mex67 crosslinking to the L1 stalk observed. In addition, we did not detect the interaction between Mex67 and the L1 stalk by yeast three hybrid (data not shown). Nevertheless, it is possible that Mex67 recruitment to the particle is enhanced by the closure of the L1 stalk, by making a binding site in the vicinity of the L1 stalk, possibly 5.8S, more accessible.

After export to the cytoplasm, the pre-60S undergoes a series of maturation events culminating in the completion of the peptidyl transferase center and release of $\mathrm{Nmd} 3$ and Tif6. We previously observed Nmd3 bound to the L1 stalk in partially closed states (Malyutin et al. 2017) and suggested that the L1 stalk may be required for the release of $\mathrm{Nmd3}$. However, the accumulation of $\mathrm{Nmd} 3$ in the nucleus in the absence of $L 1$ expression argues against a requirement for L1 in the removal of Nmd3. Similarly, mutations in $\mathrm{Nmd} 3$ that are predicted to disrupt its interaction with L1 have only a very modest impact on growth, contrary to what would be expected if the L1-Nmd3 interaction were necessary for the release of $\mathrm{Nmd} 3$ (data not shown).

\section{L1 stalk mutants in translation}

Rpl1 facilitates translation elongation assisting the release of E-site tRNAs and binding factors including elF5A (Voorhees et al. 2009; Melnikov et al. 2016). Although the mechanism of translation is highly conserved, L1 is not essential in Escherichia coli (Subramaniam and Dabbs 1980). It is essential in yeast but recent studies in both yeast and mammalian cells have detected L1 deficient ribosomes in actively translating pools (Mclntosh et al. 2011; Shi et al. 2017). It has been suggested that yeast Rpl1-deficient ribosomes associated with polysomes are strongly discriminated against during translation initiation and a fraction of them is targeted for degradation (Mclntosh et al. 2011). It has also been suggested that Rpl1 is required in "specialized ribosomes" for translating a specific subset of transcripts (Shi et al. 2017; Segev and Gerst 2018). Consistent with that, we show that ribosomes with truncated L1 stalk rRNA were able to engage in translation. However, the mutant ribosomes showed a strong bias toward lighter polysomes compared with wild-type ribosomes, possibly reflecting a general defect in elongation. Alternatively, ribosomes without an L1 stalk may support elongation at very low rates and induce more frequent stalling.

\section{MATERIALS AND METHODS}

\section{Strains plasmids and growth media}

Saccharomyces cerevisiae and plasmids used in this study are listed in Tables 1 and 2 . All cells were grown at $30^{\circ} \mathrm{C}$ in rich media (yeast extract and peptone) or synthetic dropout medium supplemented with $2 \%$ glucose or $1 \%$ galactose. Strains AJY3848, AJY3849, and AJY3850 were generated by genomic integration of TIF6-GFP-HIS3MX, ARX1-GFP-HIS3MX, and MRT4-GFPHIS3MX, amplified from AJY2909, AJY1948, and AJY3040, respectively, into KBM20. AJY4060 was generated by sporulation of KBM20 after mating with AJY1705. Strains AJY4001, AJY4008, and AJY4009 were generated by genomic integration of NMD3-TAP-HIS3MX amplified from AJY1874 into AJY3373, KBM13, and KBM20, respectively. AJY4012 and AJY4013 were generated by genomic integration of ARX1-TAP-HIS3MX amplified from AJY2491 into KBM13 and KBM20, respectively.

\section{Affinity purification of Nmd3-TAP and Arx1-TAP particles}

Cultures of strains AJY1874, AJY4001, AJY4008, AJY4009, AJY4012, and AJY4013 were grown to $O_{600}$ of 0.3 in $500 \mathrm{~mL}$ of YP media supplemented with $1 \%$ galactose. Glucose was added to a final $2 \%(w / v)$ concentration and cells were grown for $1 \mathrm{~h}$, harvested and cell pellets were frozen at $-80^{\circ} \mathrm{C}$. Cell pellets were washed and resuspended in 1.5 volumes of lysis buffer $(20 \mathrm{mM}$ HEPES, pH 7.5, 10 mM MgCl, 100 mM KCl, 5 mM $\beta$-mercaptoethanol, $1 \mathrm{mM}$ phenylmethylsulfonyl fluoride [PMSF], $1 \mu \mathrm{M}$ leupeptin, and $1 \mu \mathrm{M}$ pepstatin). Extracts were prepared by glass bead lysis and clarified by centrifugation at $4^{\circ} \mathrm{C}$ for $15 \mathrm{~min}$ at $18,000 \mathrm{~g}$. NP-40 was added to a final concentration of $0.15 \%(\mathrm{v} / \mathrm{v})$ to the clarified extract which was then incubated with rabbit lgG (Sigma-Aldrich) coupled Dynabeads (Invitrogen) for $1 \mathrm{~h}$ at $4^{\circ} \mathrm{C}$. The Dynabeads were prepared as previously described (Oeffinger et al. 2007). Beads were then washed thrice with lysis buffer containing $0.15 \% \mathrm{NP}-40$ at $4^{\circ} \mathrm{C}$ for $5 \mathrm{~min}$ each time. The bound complexes were enzymatically eluted with tobacco etch 
TABLE 1. Strains used in this study

\begin{tabular}{|c|c|c|}
\hline Strain & Genotype & Source \\
\hline AJY1185 & $\begin{array}{l}\text { MATa ade2-1 ura3-1 leu2-3 his3-11 can 1-100 rdnaA::HIS3 with pAJ724 (35S URA3 2 } \mu \text { ) and } \\
\text { pAJ719 (5S TRP 2 } \mu \text { ) }\end{array}$ & (Smith et al. 2001) \\
\hline AJY1548 & 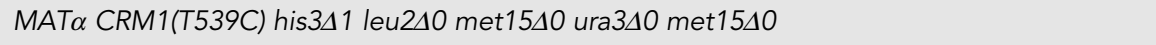 & (Hedges et al. 2005) \\
\hline AJY1705 & 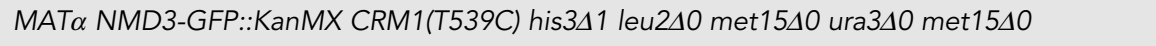 & (Hedges et al. 2005) \\
\hline AJY1874 & 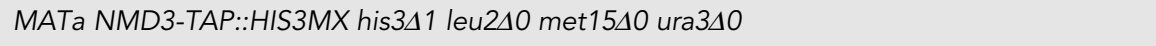 & [OpenBiosystems] \\
\hline AJY1948 & 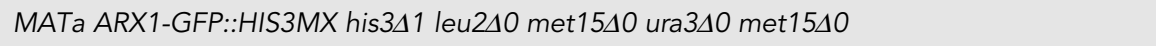 & [OpenBiosystems] \\
\hline AJY2491 & 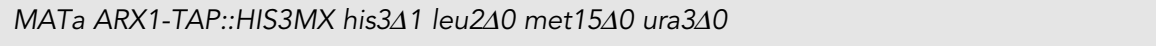 & [OpenBiosystems] \\
\hline AJY2629 & 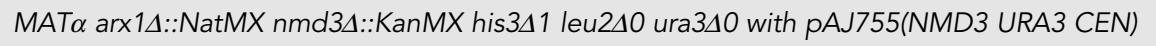 & This study \\
\hline AJY2909 & 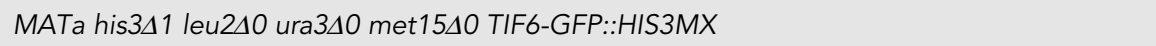 & [OpenBiosystems] \\
\hline AJY3040 & 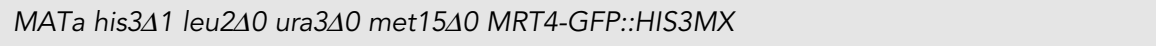 & [Open Biosystems] \\
\hline AJY3247 & 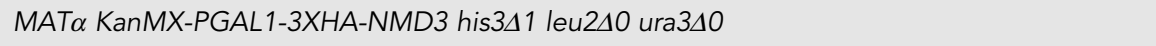 & This study \\
\hline AJY3373 & 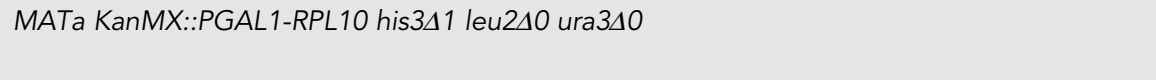 & $\begin{array}{l}\text { (De Keersmaecker et al. } \\
\text { 2013) }\end{array}$ \\
\hline AJY3848 & 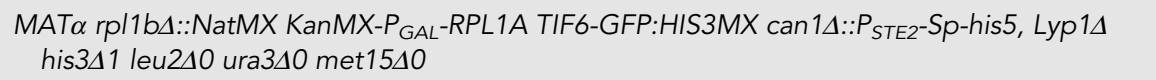 & This study \\
\hline AJY3849 & 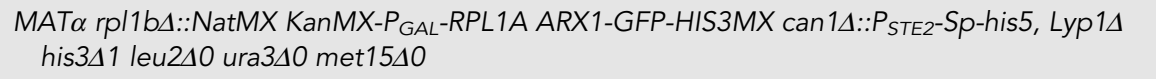 & This study \\
\hline AJY3850 & 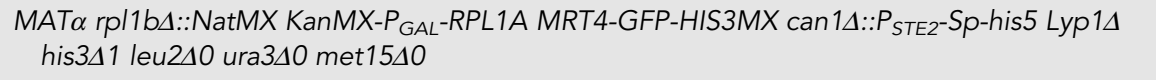 & This study \\
\hline AJY4001 & 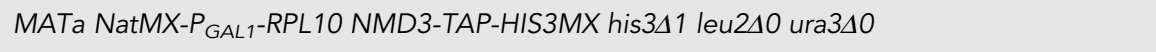 & This study \\
\hline AJY4008 & 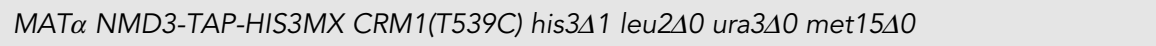 & This study \\
\hline AJY4009 & 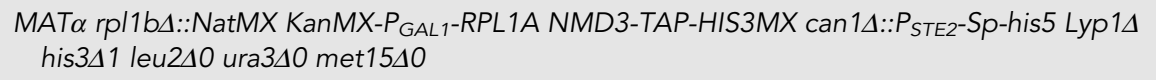 & This study \\
\hline AJY4012 & 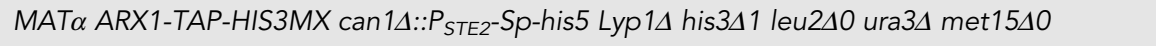 & This study \\
\hline AJY4013 & 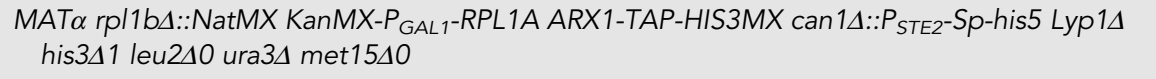 & This study \\
\hline AJY4060 & 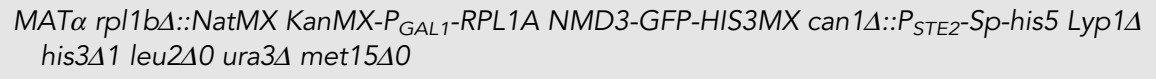 & This study \\
\hline KBM13 & 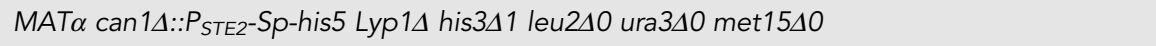 & (Mclntosh et al. 2011) \\
\hline KBM20 & 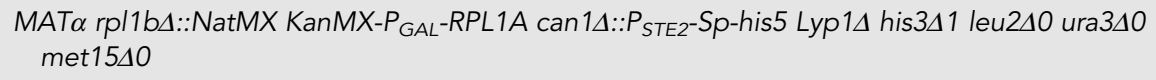 & (Mclntosh et al. 2011) \\
\hline BY4741 (WT) & MATa his3 $\Delta 1$ leu2 $2 \Delta 0$ met15 $\Delta 0$ ura3 $\Delta 0$ met15 $\Delta 0$ & \\
\hline
\end{tabular}

virus protease in lysis buffer containing $0.15 \%$ NP-40 and $1 \mathrm{mM}$ Dithiothreitol for $90 \mathrm{~min}$ at $16^{\circ} \mathrm{C}$.

\section{Polysome analysis and western blots}

Cultures of strains KBM13 and KBM20 were grown to $\mathrm{OD}_{600}$ of 0.3 in $150 \mathrm{~mL}$ of YP media supplemented with $1 \%$ galactose. Glucose was added to a final $2 \%(w / v)$ concentration and cells were grown for two more hours. Cycloheximide (CHX) was added to a final concentration of $100 \mu \mathrm{g} / \mathrm{mL}$, cultures incubated for $10 \mathrm{~min}$ at $30^{\circ} \mathrm{C}$ to arrest translation and preserve polysomes and cells were harvested and frozen at $-80^{\circ} \mathrm{C}$. Cell pellets were washed and resuspended in 1.5 volumes of polysome lysis buffer (20 mM HEPES, pH 7.5, 10 mM MgCl $, 100 \mathrm{mM} \mathrm{KCl}, 100 \mu \mathrm{g} / \mathrm{mL}$ $\mathrm{CHX}, 5 \mathrm{mM} \beta$-mercaptoethanol, 1 mM PMSF, $1 \mu \mathrm{M}$ leupeptin, and $1 \mu \mathrm{M}$ pepstatin). Extracts were prepared by glass bead lysis and clarified by centrifugation at $4^{\circ} \mathrm{C}$ for $15 \mathrm{~min}$ at $18,000 \mathrm{~g}$. Nine $A_{260}$ units of clarified extract were loaded onto $7 \%-47 \%$ sucrose gradients prepared in polysome lysis buffer and
TABLE 2. Plasmids used in this study

\begin{tabular}{lll}
\hline Plasmid & \multicolumn{1}{c}{ Description } & \multicolumn{1}{c}{ Source } \\
\hline pAJ718 & rDNA LEU2 $2 \mu$ & (White et al. 2008) \\
pAJ719 & 5S rDNA LEU2 $2 \mu$ & (Smith et al. 2001) \\
pAJ724 & rDNA URA3 $2 \mu$ & (Smith et al. 2001) \\
pAJ582 & NMD3-GFP LEU2 CEN & (Hedges et al. 2005) \\
pAJ907 & RPL25-GFP LEU2 CEN & (Kallstrom et al. 2003) \\
pAJ1181 & rDNA LEU2 CEN & This study \\
pAJ3605 & AL1 stalk-rDNA LEU2 CEN & This study \\
pAJ3972 & MEX67 MTR2 URA3 2 $\mu$ & This study \\
pAJ4315 & ARX1 URA3 2 $\mu$ & This study \\
pAJ4316 & BUD20 URA3 2 $\mu$ & This study \\
pAJ4338 & SIK1-mRFP URA3 2 $\mu$ & This study \\
pRS415 & LEU2 CEN & \\
pRS416 & URA3 CEN & \\
pRS426 & URA3 $2 \mu$ & \\
\hline
\end{tabular}


TABLE 3. Oligonucleotides used in this study

\begin{tabular}{ll}
\hline Oligo & \multicolumn{1}{c}{ Sequence } \\
\hline AJO190 & 5'-GTCTGGACCTGGTGAGTTTCCC-3' \\
AJO192 & 5'-CCCGCCGTTTACCCGCGCTTGG-3' \\
AJO628 & 5'-CTGCAGAAGAACCGGAGTGCAATGGCTCTTC-3' \\
AJO1247 & 5'-Cy3TCGGGCCTGCAGAAGAACCGGAGTGCAATGGCTCTTCACCGA-3' \\
\hline
\end{tabular}

centrifuged for $2.5 \mathrm{~h}$ at 40,000 rpm in a Beckman SW40 rotor. Gradients were fractionated using an ISCO Model 640 fractionator into $600 \mu \mathrm{L}$ fractions with continuous monitoring at 254 $\mathrm{nm}$. To each fraction, $1.2 \mathrm{~mL}$ of $100 \%$ ethanol was added, vortexed and stored at $-20^{\circ} \mathrm{C}$ overnight. Fractions were centrifuged at $4^{\circ} \mathrm{C}$ for $15 \mathrm{~min}$ at $18,000 \mathrm{~g}$ and pellets were dissolved in $1 \times$ Laemmli buffer and heated at $99^{\circ} \mathrm{C}$ for 3 min. Proteins were separated on $6 \%-15 \%$ gradient SDS-PAGE gels, transferred to nitrocellulose membrane and subjected to western blot analysis using anti-Nmd3, anti-Rpl8 (K.-Y. Lo), and anti-Rpl1 (J. Warner) antisera.

\section{Sucrose gradient sedimentation and northern blot analyses}

Saturated cultures of BY4741 transformed with pAJ1181 or pAJ3605 were diluted to $\mathrm{OD}_{600}$ of 0.1 in SD Leu ${ }^{-}$and grown to mid-log phase. Cell cultures were treated with $\mathrm{CHX}$ at $50 \mu \mathrm{g} / \mathrm{mL}$ for $10 \mathrm{~min}$ at $30^{\circ} \mathrm{C}$ to inhibit translation, and then cells were harvested and stored at $-80^{\circ} \mathrm{C}$. Cells were washed once and then resuspended in 1.5-2 volumes lysis buffer $(50 \mathrm{mM}$ Tris- $\mathrm{HCl}$ $\mathrm{pH} 7.5,100 \mathrm{mM} \mathrm{KCl}, 5 \mathrm{mM} \mathrm{MgCl} 2,50 \mu \mathrm{g} / \mathrm{mL} \mathrm{CHX}, 1 \mathrm{mM}$ PMSF, benzamidine, and $1 \mu \mathrm{M}$ each of leupeptin and pepstatin). Extracts were prepared by glass bead lysis and clarified by centrifugation at $4^{\circ} \mathrm{C}$ for $15 \mathrm{~min}$ at $18,000 \mathrm{~g}$. Nine $A_{260}$ units of clarified extract were applied to sucrose density gradients and fractionated as described above. Sixty microliters of $20 \%$ SDS, $60 \mu \mathrm{L}$ of $3 \mathrm{M}$ Sodium acetate $\mathrm{pH} 5.2$ and $1.3 \mathrm{~mL} 100 \%$ ethanol were added to each sample and nucleic acids were precipitated overnight at $-20^{\circ} \mathrm{C}$. The precipitate was pelleted by centrifugation for $30 \mathrm{~min}$ at $18,000 \mathrm{rpm}$ and $4^{\circ} \mathrm{C}$. RNA pellets were washed with $70 \%$ ethanol and air dried. Pellets from each fraction were dissolved in $50 \mu \mathrm{L}$ RNase free water. Total RNA from one $A_{260}$ unit of clarified lysate was extracted similarly and dissolved in $50 \mu \mathrm{L}$ RNase free water. Ten microliters of RNA samples from lysate and from sucrose gradient fractions 1-19 were vacuum dried and dissolved in $10 \mu \mathrm{L}$ RNA sample loading buffer (Invitrogen AM8552). RNAs were resolved by electrophoresis through 1.2\%agarose MOPS $6 \%$ formaldehyde gel for $4 \mathrm{~h}$ at $50 \mathrm{~V}$. Northern blotting was performed as previously described (Li et al. 2009) using the oligos AJO190, AJO192, and AJO628 (Table 3), and the signal was detected by phosphoimaging on a GE Typhoon FLA9500.

\section{Microscopy}

For direct fluorescence experiments, cells were grown in selective medium ( $\mathrm{Leu}^{-}$or $\mathrm{His}^{-}$) supplemented with $1 \%$ galactose for $48 \mathrm{~h}$, then diluted fourfold in medium containing $2 \%$ glucose and grown for 60-90 $\mathrm{min}$ to repress the expression of RPL1A.
Images were captured using a Nikon E800 microscope fitted with a $100 \times$ Plan Apo objective and a Photometrics CoolSNAP ES camera controlled by NIS-Elements software. For FISH experiments, BY4741 cells transformed with pAJ1181 or pAJ3605 were grown to saturation in $\mathrm{Leu}^{-}$medium with $2 \%$ glucose, then diluted fivefold in fresh $\mathrm{Leu}^{-}$glucose medium and continued to grow for $60 \mathrm{~min}$. Formaldehyde was added to a final concentration of $4.5 \%$ to the cell cultures and cells were fixed by agitating gently at room temperature for $30 \mathrm{~min}$. Fixed cells were pelleted and washed twice with KSorb buffer (1.2 M sorbitol, $0.1 \mathrm{M}$ potassium phosphate buffer 7.0). Cell pellets resuspended in $200 \mu \mathrm{L} \mathrm{KSorb,}$ were treated with $50 \mu \mathrm{g} / \mathrm{mL}$ Zymolyase T20 for 15 min at $37^{\circ} \mathrm{C}$ in the presence of $20 \mathrm{mM}$ Vanadyl Ribonucleoside complex (VRC), $28 \mathrm{mM} \beta$-mercaptoethanol and $1 \mathrm{mM}$ PMSF. Cells were gently pelleted and washed with ice cold KSorb buffer thrice and resuspended in $100 \mu \mathrm{L}$ Ksorb buffer. Thirty-five microliter cell suspension was applied to the wells of Teflon-coated Immunofluorescence slides (Polysciences Inc., No. 18357) precoated with Poly-lysine. Slides were incubated in a moist chamber at room temperature for $10 \mathrm{~min}$, then excess cells were gently aspirated, and the slides were stored in $70 \%$ ethanol at $-20^{\circ} \mathrm{C}$. Cells were rehydrated by washing twice with $2 \times$ SSC $(300 \mathrm{mM} \mathrm{NaCl}$, $30 \mathrm{mM}$ Sodium Citrate $\mathrm{pH}$ 7.0) and then incubated in $40 \mu \mathrm{L}$ Prehybridization solution (10\% Dextran sulfate, 50\% deionized formamide, $1 \times$ Denhardt's, $2 \mathrm{mM}$ VRC and $4 \times$ SSC, $0.2 \%$ BSA, $25 \mu \mathrm{g}$ yeast tRNA and $500 \mu \mathrm{g} / \mathrm{mL}$ ssDNA) for $1 \mathrm{~h}$ at $72^{\circ} \mathrm{C}$ in a moist chamber. Excess solution was removed by aspiration and replaced with $40 \mu \mathrm{L}$ of Hybridization solution (Prehybridization solution containing $1 \mu \mathrm{M}$ Cy3 labeled oligo AJO1247) in each well. The slide was incubated in a moist chamber at $72^{\circ} \mathrm{C}$ for $1 \mathrm{~h}$ followed by overnight incubation at $37^{\circ} \mathrm{C}$. The next day, the wells were washed with $2 \times$ SSC and then $1 \times$ SSC containing $0.1 \%$ NP-40 for $30 \mathrm{~min}$ each. Cells were incubated for $2 \mathrm{~min}$ with $1 \mu \mathrm{g} / \mathrm{mL}$ DAPI, washed twice with PBS and mounted in AquaPoly/Mount (Polysciences, Inc.). Images were captured as described above.

\section{ACKNOWLEDGMENTS}

We thank Dr. J.R. Warner (Albert Einstein College of Medicine, New York) for his generous gift of yeast strains and anti-Rpl1 antibodies, Dr. V. Panse (ETH Zurich) for anti-Mex67 antibody and Dr. K.-Y. Lo for anti-Rpl8 antibody and members of the Johnson laboratory for helpful discussions. This work was supported by the National Institutes of Health (NIH) Grants GM53655 and GM127127 (to A.W.J.).

Author contributions: S.M. and A.W.J. designed the study. S.M. designed and performed the experiments. J.J.B. performed the 
northern blot analysis. S.M. and A.W.J. interpreted the results and wrote the manuscript.

Received May 8, 2019; accepted August 8, 2019.

\section{REFERENCES}

Altvater M, Chang Y, Melnik A, Occhipinti L, Schütz S, Rothenbusch U, Picotti P, Panse VG. 2012. Targeted proteomics reveals compositional dynamics of $60 \mathrm{~S}$ pre-ribosomes after nuclear export. Mol Syst Biol 8: 628. doi:10.1038/msb.2012.63

Amsterdam A, Sadler KC, Lai K, Farrington S, Bronson RT, Lees JA, Hopkins N. 2004. Many ribosomal protein genes are cancer genes in Zebrafish. PLoS Biol 2: e139. doi:10.1371/journal.pbio .0020139

Barrio-Garcia C, Thoms M, Flemming D, Kater L, Berninghausen O, Baßler J, Beckmann R, Hurt E. 2016. Architecture of the Rix1Rea1 checkpoint machinery during pre-60S-ribosome remodeling. Nat Struct Mol Biol 23: 37-44. doi:10.1038/nsmb.3132

Bassler J, Klein I, Schmidt C, Kallas M, Thomson E, Wagner MA, Bradatsch B, Rechberger G, Strohmaier H, Hurt E, et al. 2012. The conserved Bud20 zinc finger protein is a new component of the ribosomal 60S subunit export machinery. Mol Cell Biol 32: 4898-4912. doi:10.1128/MCB.00910-12

Ben-Shem A, Garreau de Loubresse N, Melnikov S, Jenner L, Yusupova G, Yusupov M. 2011. The structure of the eukaryotic ribosome at $3.0 \AA$ resolution. Science 334: 1524-1529. doi:10 $.1126 /$ science. 1212642

Boulon S, Westman BJ, Hutten S, Boisvert F-M, Lamond Al. 2010. The nucleolus under stress. Mol Cell 40: 216-227. doi:10.1016/j .molcel.2010.09.024

Bradatsch B, Katahira J, Kowalinski E, Bange G, Yao W, Sekimoto T, Baumgärtel V, Boese G, Bassler J, Wild K, et al. 2007. Arx1 functions as an unorthodox nuclear export receptor for the 605 preribosomal subunit. Mol Cell 27: 767-779. doi:10.1016/j.molcel .2007.06.034

Cheng Z, Mugler CF, Keskin A, Hodapp S, Chan LY-L, Weis K, Mertins P, Regev A, Jovanovic M, Brar GA. 2019. Small and large ribosomal subunit deficiencies lead to distinct gene expression signatures that reflect cellular growth rate. Mol Cell 73: 36-47. e10. doi:10.1016/j.molcel.2018.10.032

Correll CC, Wool IG, Munishkin A. 1999. The two faces of the Escherichia coli $23 \mathrm{~S}$ rRNA sarcin/ricin domain: the structure at 1.11 Å resolution. J Mol Biol 292: 275-287. doi:10.1006/jmbi .1999 .3072

De Keersmaecker K, Atak ZK, Li N, Vicente C, Patchett S, Girardi T, Gianfelici V, Geerdens E, Clappier E, Porcu M, et al. 2013. Exome sequencing identifies mutation in CNOT3 and ribosomal genes RPL5 and RPL10 in T-cell acute lymphoblastic leukemia. Nat Genet 45: 186-190. doi:10.1038/ng.2508

Gadal O, Strau D, Kessl J, Trumpower B, Tollervey D, Hurt E. 2001. Nuclear export of 60S ribosomal subunits depends on Xpo1p and requires a nuclear export sequence-containing factor, Nmd3p, that associates with the large subunit protein Rpl10p. Mol Cell Biol 21: 3405-3415. doi:10.1128/MCB.21.10.3405-3415.2001

Greber BJ, Boehringer D, Montellese C, Ban N. 2012. Cryo-EM structures of Arx1 and maturation factors Rei1 and Jjj1 bound to the $60 \mathrm{~S}$ ribosomal subunit. Nat Struct Mol Biol 19: 1228-1233. doi:10 $.1038 / \mathrm{nsmb} .2425$

Greber BJ, Gerhardy S, Leitner A, Leibundgut M, Salem M, Boehringer D, Leulliot N, Aebersold R, Panse VG, Ban N. 2016. Insertion of the biogenesis factor rei1 probes the ribosomal tunnel during 60S maturation. Cell 164: 91-102. doi:10.1016/j.cell.2015 .11 .027
Grosshans H, Deinert K, Hurt E, Simos G. 2001. Biogenesis of the signal recognition particle (SRP) involves import of SRP proteins into the nucleolus, assembly with the SRP-RNA, and Xpo1p-mediated export. J Cell Biol 153: 745-762. doi:10.1083/jcb.153.4.745

Hedges J, West M, Johnson AW. 2005. Release of the export adapter, Nmd3p, from the 60S ribosomal subunit requires Rpl10p and the cytoplasmic GTPase Lsg1p. EMBO J 24: 567-579. doi:10.1038/sj .emboj.7600547

Ho JH-N, Kallstrom G, Johnson AW. 2000. Nmd3p is a Crm1p-dependent adapter protein for nuclear export of the large ribosomal subunit. J Cell Biol 151: 1057-1066. doi:10.1083/jcb.151.5.1057

Hung N-J, Lo K-Y, Patel SS, Helmke K, Johnson AW. 2008. Arx1 is a nuclear export receptor for the 60s ribosomal subunit in yeast. Mol Biol Cell 19: 735-744. doi:10.1091/mbc.e07-09-0968

Kallstrom G, Hedges J, Johnson A. 2003. The putative GTPases Nog1 $p$ and Lsg1 $p$ are required for 605 ribosomal subunit biogenesis and are localized to the nucleus and cytoplasm, respectively. Mol Cell Bio 23: 4344-4355. doi:10.1128/mcb.23.12.4344-4355 .2003

Kargas V, Castro-Hartmann P, Escudero-Urquijo N, Dent K, Hilcenko C, Sailer C, Zisser G, Marques-Carvalho MJ, Pellegrino S, Wawiórka $L$, et al. 2019. Mechanism of completion of peptidyltransferase centre assembly in eukaryotes. eLife 8: e44904. doi:10 $.7554 /$ eLife.44904

Kemmler S, Occhipinti L, Veisu M, Panse VG. 2009. Yvh1 is required for a late maturation step in the 60S biogenesis pathway. J Cell Biol 186: 863-880. doi:10.1083/jcb.200904111

Kressler D, Hurt E, Baßler J. 2017. A puzzle of life: crafting ribosomal subunits. Trends Biochem Sci 42: 640-654. doi:10.1016/j.tibs .2017.05.005

Li Z, Lee I, Moradi E, Hung NJ, Johnson AW, Marcotte EM. 2009. Rational extension of the ribosome biogenesis pathway using network-guided genetics. PLoS Biol 7: e1000213. doi:10.1371/jour nal.pbio.1000213

Lo K-Y, Johnson AW. 2009. Reengineering ribosome export. Mol Biol Cell 20: 1545-1554. doi:10.1091/mbc.e08-10-1000

Lo KY, Li Z, Wang F, Marcotte EM, Johnson AW. 2009. Ribosome stalk assembly requires the dual-specificity phosphatase Yvh1 for the exchange of Mrt4 with PO. J Cell Biol 186: 849-862. doi:10 $.1083 / j c b .200904110$

Lo K-Y, Li Z, Bussiere C, Bresson S, Marcotte EM, Johnson AW. 2010. Defining the pathway of cytoplasmic maturation of the $60 \mathrm{~S}$ ribosomal subunit. Mol Cell 39: 196-208. doi:10.1016/j.molcel.2010 .06 .018

Ma C, Wu S, Li N, Chen Y, Yan K, Li Z, Zheng L, Lei J, Woolford JL, Gao N. 2017. Structural snapshot of cytoplasmic pre-60S ribosomal particles bound by Nmd3, Lsg1, Tif6 and Reh1. Nat Struct Mol Biol 24: 214-220. doi:10.1038/nsmb.3364

Malyutin AG, Musalgaonkar S, Patchett S, Frank J, Johnson AW. 2017. $\mathrm{Nmd} 3$ is a structural mimic of elF5A, and activates the cpGTPase Lsg1 during 60S ribosome biogenesis. EMBO J 36: 854-868. doi:10.15252/embj.201696012

Matsuo Y, Granneman S, Thoms M, Manikas R-G, Tollervey D, Hurt E. 2014. Coupled GTPase and remodelling ATPase activities form a checkpoint for ribosome export. Nature 505: 112-116. doi:10 .1038 /nature12731

Mclntosh KB, Bhattacharya A, Willis IM, Warner JR. 2011. Eukaryotic cells producing ribosomes deficient in Rpl1 are hypersensitive to defects in the ubiquitin-proteasome system. PLoS One 6: e23579. doi:10.1371/journal.pone.0023579

Melnikov S, Mailliot J, Shin BS, Rigger L, Yusupova G, Micura R, Dever TE, Yusupov M. 2016. Crystal structure of hypusine-containing translation factor elF5A bound to a rotated eukaryotic ribosome. J Mol Biol 428: 3570-3576. doi:10.1016/j.jmb.2016.05.011 
Mills EW, Green R. 2017. Ribosomopathies: there's strength in numbers. Science 358: eaan2755. doi:10.1126/science.aan2755

Nerurkar P, Gillet L, Pena C, Schubert OT, Altvater M, Chang Y, Aebersold R, Panse V. 2018. The GTPase Nog1 couples polypeptide exit tunnel quality control with ribosomal stalk assembly. bioRxiv. doi:10.1101/462333

Oeffinger M, Wei KE, Rogers R, DeGrasse JA, Chait BT, Aitchison JD, Rout MP. 2007. Comprehensive analysis of diverse ribonucleoprotein complexes. Nat Methods 4: 951-956. doi:10.1038/nmeth1101

Peña C, Hurt E, Panse VG. 2017. Eukaryotic ribosome assembly, transport and quality control. Nat Struct Mol Biol 24: 689-699. doi:10 .1038/nsmb.3454

Ribbeck K, Görlich D. 2002. The permeability barrier of nuclear pore complexes appears to operate via hydrophobic exclusion. EMBO J 21: 2664-2671. doi:10.1093/emboj/21.11.2664

Rodríguez-Galán O, García-Gómez JJ, Kressler D, de la Cruz J. 2015. Immature large ribosomal subunits containing the 7S pre-rRNA can engage in translation in Saccharomyces cerevisiae. RNA Biol 12: 838-846. doi:10.1080/15476286.2015.1058477

Sardana R, Liu X, Granneman S, Zhu J, Gill M, Papoulas O, Marcotte EM, Tollervey D, Correll CC, Johnson AW. 2015. The DEAH-box Helicase Dhr1 dissociates U3 from the pre-rRNA to promote formation of the central pseudoknot. Biology 13: e1002083. doi:10 .1371/journal.pbio.1002083

Sarkar A, Pech M, Thoms M, Beckmann R, Hurt E. 2016. Ribosomestalk biogenesis is coupled with recruitment of nuclear-export factor to the nascent 60S subunit. Nat Struct Mol Biol 23: 1074-1082. doi:10.1038/nsmb.3312

Sarkar A, Thoms M, Barrio-Garcia C, Thomson E, Flemming D, Beckmann R, Hurt E. 2017. Preribosomes escaping from the nucleus are caught during translation by cytoplasmic quality control. Nat Struct Mol Biol 24: 1107-1115. doi:10.1038/nsmb.3495

Segev N, Gerst JE. 2018. Specialized ribosomes and specific ribosomal protein paralogs control translation of mitochondrial proteins. $J$ Cell Biol 217: 117-126. doi:10.1083/jcb.201706059

Shi Z, Fujii K, Kovary KM, Genuth NR, Röst HL, Teruel MN, Barna M. 2017. Heterogeneous ribosomes preferentially translate distinct subpools of mRNAs genome-wide. Mol Cell 67: 71-83.e7. doi:10.1016/j.molcel.2017.05.021
Smith MW, Meskauskas A, Wang P, Sergiev PV, Dinman JD. 2001. Saturation mutagenesis of $5 \mathrm{~S}$ rRNA in Saccharomyces cerevisiae. Mol Cell Biol 21: 8264-8275. doi:10.1128/MCB.21.24.82648275.2001

Subramaniam AR, Dabbs ER. 1980. Functional studies on ribosomes lacking protein L1 from mutant Escherichia coli. Eur J Biochem 112: 425-430. doi:10.1111/j.1432-1033.1980.tb07222.x

Thomas F, Kutay U. 2003. Biogenesis and nuclear export of ribosomal subunits in higher eukaryotes depend on the CRM1 export pathway. J Cell Sci 116: 2409-2419. doi:10.1242/jcs.00464

Tuck AC, Tollervey D. 2013. A transcriptome-wide atlas of RNP composition reveals diverse classes of mRNAs and IncRNAs. Cell 154: 996-1009. doi:10.1016/j.cell.2013.07.047

Voorhees RM, Weixlbaumer A, Loakes D, Kelley AC, Ramakrishnan V. 2009. Insights into substrate stabilization from snapshots of the peptidyl transferase center of the intact $70 \mathrm{~S}$ ribosome. Nat Struct Mol Biol 16: 528-533. doi:10.1038/nsmb.1577

Warren AJ. 2018. Molecular basis of the human ribosomopathy Shwachman-Diamond syndrome. Adv Biol Regul 67: 109-127. doi:10.1016/j.jbior.2017.09.002

White J, Li Z, Sardana R, Bujnicki JM, Marcotte EM, Johnson AW. 2008. Bud23 methylates G1575 of $18 \mathrm{~S}$ rRNA and is required for efficient nuclear export of pre-40S subunits. Mol Cell Biol 28: 3151-3161. doi:10.1128/MCB.01674-07

Woolford JL, Baserga SJ. 2013. Ribosome biogenesis in the yeast Saccharomyces cerevisiae. Genetics 195: 643-681. doi:10.1534/ genetics.113.153197

Wu S, Tutuncuoglu B, Yan K, Brown H, Zhang Y, Tan D, Gamalinda M, Yuan Y, Li Z, Jakovljevic J, et al. 2016. Diverse roles of assembly factors revealed by structures of late nuclear pre-60S ribosomes. Nature 534: 133-137. doi:10.1038/nature17942

Yao W, Roser D, Köhler A, Bradatsch B, Baßler J, Hurt E. 2007. Nuclear export of ribosomal 60S subunits by the general mRNA export receptor Mex67-Mtr2. Mol Cell 26: 51-62. doi:10.1016/j.molcel .2007 .02 .018

Zhou Y, Musalgaonkar S, Johnson AW, Taylor DW. 2019. Tightlyorchestrated rearrangements govern catalytic center assembly of the ribosome. Nat Commun 10: 958. doi:10.1038/s41467-01908880-0 

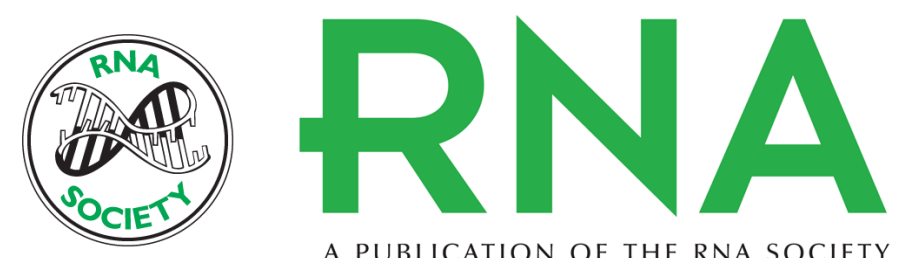

A PUBLICATION OF THE RNA SOCIETY

\title{
The L1 stalk is required for efficient export of nascent large ribosomal subunits in yeast
}

Sharmishtha Musalgaonkar, Joshua J. Black and Arlen W. Johnson

RNA 2019 25: 1549-1560 originally published online August 22, 2019

Access the most recent version at doi:10.1261/rna.071811.119

\begin{abstract}
References This article cites 52 articles, 18 of which can be accessed free at: http://rnajournal.cshlp.org/content/25/11/1549.full.html\#ref-list-1

Creative This article is distributed exclusively by the RNA Society for the first 12 months after the Commons full-issue publication date (see http://rnajournal.cshlp.org/site/misc/terms.xhtml). After 12 License months, it is available under a Creative Commons License (Attribution-NonCommercial 4.0 International), as described at http://creativecommons.org/licenses/by-nc/4.0/.
\end{abstract}

Email Alerting Receive free email alerts when new articles cite this article - sign up in the box at the Service top right corner of the article or click here. 